\title{
Gibbs states of lattice spin systems with unbounded disorder|*
}

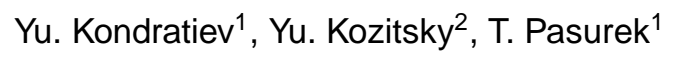 \\ ${ }^{1}$ Fakultät für Mathematik, Universität Bielefeld, D-33615 Bielefeld, Germany \\ 2 Instytut Matematyki, Uniwersytet Marii Curie-Skłodowskiej, 20-031 Lublin, Poland
}

Received September 9, 2010, in final form September 28, 2010

The Gibbs states of a spin system on the lattice $\mathbb{Z}^{d}$ with pair interactions $J_{x y} \sigma(x) \sigma(y)$ are studied. Here $\langle x, y\rangle \in$ E, i.e. $x$ and $y$ are neighbors in $\mathbb{Z}^{d}$. The intensities $J_{x y}$ and the spins $\sigma(x), \sigma(y)$ are arbitrarily real. To control their growth we introduce appropriate sets $\mathcal{J}_{q} \subset \mathbb{R}^{\mathrm{E}}$ and $\mathcal{S}_{p} \subset \mathbb{R}^{\mathbb{Z}^{d}}$ and show that, for every $J=\left(J_{x y}\right) \in \mathcal{J}_{q}:(\mathrm{a})$ the set of Gibbs states $\mathcal{G}_{p}(J)=\left\{\mu\right.$ : solves DLR, $\left.\mu\left(\mathcal{S}_{p}\right)=1\right\}$ is non-void and weakly compact; (b) each $\mu \in \mathcal{G}_{p}(J)$ obeys an integrability estimate, the same for all $\mu$. Next we study the case where $\mathcal{J}_{q}$ is equipped with a norm, with the Borel $\sigma$-field $\mathcal{B}\left(\mathcal{J}_{q}\right)$, and with a complete probability measure $\nu$. We show that the set-valued map $\mathcal{J}_{q} \ni J \mapsto \mathcal{G}_{p}(J)$ has measurable selections $\mathcal{J}_{q} \ni J \mapsto \mu(J) \in \mathcal{G}_{p}(J)$, which are random Gibbs measures. We demonstrate that the empirical distributions $N^{-1} \sum_{n=1}^{N} \pi_{\Delta_{n}}(\cdot \mid J, \xi)$, obtained from the local conditional Gibbs measures $\pi_{\Delta_{n}}(\cdot \mid J, \xi)$ and from exhausting sequences of $\Delta_{n} \subset \mathbb{Z}^{d}$, have $\nu$-a.s. weak limits as $N \rightarrow+\infty$, which are random Gibbs measures. Similarly, we show the existence of the $\nu$-a.s. weak limits of the empirical metastates $N^{-1} \sum_{n=1}^{N} \delta_{\pi_{\Delta_{n}}(\cdot \mid J, \xi)}$, which are Aizenman-Wehr metastates. Finally, we demonstrate that the limiting thermodynamic pressure exists under some further conditions on $\nu$.

Key words: Aizenman-Wehr metastate, Newman-Stein empirical metastate, chaotic size dependence, Komlós theorem, quenched pressure, spin glass

PACS: $61.43 . F s, 64.60 . D e, 64.70 . \mathrm{kj}$

\section{Introduction}

In the present note, we announce a number of the results describing Gibbs states of a lattice system with unbounded spins and unbounded random interactions. Their complete proof will be published in a separate work. Our motivations, as well as the discussion of the connections of our results with those known for spin models with random interactions are given in section 3.2 below.

Throughout the note, for a topological space $\mathcal{S}$, by $\mathcal{P}(\mathcal{S})$ we denote the set of all probability measures on $(\mathcal{S}, \mathcal{B}(\mathcal{S})$ ), where $\mathcal{B}(\mathcal{S})$ will always stand for the corresponding Borel $\sigma$-field.

Given a countable set $\mathrm{X}$, a random field on $\mathrm{X}$ is a collection of random variables - spins, defined on some probability space and taking values in the corresponding single-spin (Polish) spaces $S_{x}$, $x \in \mathrm{X}$. In a 'canonical version', the probability space is $(\mathcal{S}, \mathcal{B}(\mathcal{S}), \mu)$, where $\mathcal{S}$ is the product space of all $S_{x}$. Then the notion random field is attributed to the latter measure as well. A particular case of such a field is the product measure of some single-spin probability measures $\chi_{x}, x \in \mathrm{X}$. Gibbs random fields with pair interactions are constructed as perturbations of $\prod_{x \in \mathrm{X}} \chi_{x}$ by the 'densities'

$$
\exp \left(\sum_{\langle x, y\rangle} W_{x y}(\sigma(x), \sigma(y))\right)
$$

where $W_{x y}: S_{x} \times S_{y} \rightarrow \mathbb{R}$ are measurable functions - interaction potentials, whereas the sum is taken over a subset of $X \times X$. Such a field defines the graph $G=(X, E)$, where the set of edges $\mathrm{E}$ consists of those pairs $\{x, y\}$ where $W_{x y}$ is not the zero function. The case of a special

*Supported by the DFG through SFB 701: "Spektrale Strukturen und Topologische Methoden in der Mathematik" and through the research project 436 POL 125/0-1. 
interest is where the potentials are random. Then one deals with another random field, this time on $\mathrm{E}$, represented by the triple $(\mathcal{W}, \mathcal{F}, P)$. Here $\mathcal{W}$ is the space of interactions consisting of $W=$ $\left(W_{x y}\right)_{\langle x, y\rangle \in \mathrm{E}}, \mathcal{F}$ is an appropriate $\sigma$-field, and $P$ is a probability measure. A standard assumption is that the degree of each vertex is finite and that the functions $W_{x y}: S_{x} \times S_{y} \rightarrow \mathbb{R}$ are $P$-almost surely bounded, in which case the interactions are called regular, c.f. Definition 6.2.1 in [1], page 99. The only irregular case studied in the literature is that of the long-range spin glasses, where the single-spin spaces are finite, and thus the functions $W_{x y}$ are bounded, but the vertex degrees are infinite. In the case of regular $W$, a measurable map

$$
\mathcal{W} \ni W \mapsto \mu(W) \in \mathcal{P}(\mathcal{S})
$$

is called a random Gibbs measure, or a random Gibbs state, if for P-almost all $W, \mu(W)$ has a Markov property, standard for Gibbs measures, c.f. Definition 6.2.5 in [1]. The measurability in (1.2) is the key point since only in this case one can speak about averages with respect to the disorder, that is, about the expectations $\mathbb{E}_{P} \Phi\left(\mathbb{E}_{\mu(W)} F\right)$, where $F: \mathcal{S} \rightarrow \mathbb{R}$ and $\Phi: \mathbb{R} \rightarrow \mathbb{R}$ are appropriate functions, see the discussion in section 6.2 in [1]. In general, for models with the interactions (1.1), there might exist multiple Gibbs measures 1 . Hence, the map $W \mapsto\{\mu(W): \mu(W)$ is a Gibbs measure $\}$ can be set-valued and the existence of its measurable selections (1.2) is not obvious. To the best of our knowledge, in a systematic way this aspect of the theory has never been discussed so far. Thus, one of the aims of this work is to look at the problem of Gibbs fields with random interactions from the point of view of the set-valued analysis [2]. Another aim is to elaborate a method, which would allow us to study the models with unbounded interactions - the other irregular case that has not been studied yet. In order to make the things as much transparent as possible, we consider the simplest case where the graph is a lattice $\mathbb{Z}^{d}$ with the edge set $\mathrm{E}=\{(x, y):|x-y|=1\}$, whereas the interaction potentials have the form

$$
W_{x y}(u, v)=J_{x y} u v, \quad J_{x y}, u, v \in \mathbb{R}
$$

that is, all $S_{x}$ are the copies of $\mathbb{R}$. In the physical terminology, this is a lattice spin model with unbounded spins and a harmonic pair interaction. The existence and the properties of the corresponding Gibbs fields for all $J_{x y}$ being the same (or just uniformly bounded) and nonrandom have been studied since the 1970th, see [3, 44] and the bibliographic notes in [5]. However, the case of $\sup _{\langle x, y\rangle \in \mathrm{E}}\left|J_{x y}\right|=+\infty$ has not been studied so far. To control the growth of $J=\left(J_{x y}\right)_{\langle x, y\rangle \in \mathrm{E}}$ and $\sigma=(\sigma(x))_{x \in \mathrm{X}}$, we introduce two Banach spaces $\mathcal{J}_{q} \subset \mathbb{R}^{\mathrm{E}}$ and $\mathcal{S}_{p} \subset \mathbb{R}^{\mathbb{Z}^{d}}$. They are large enough so that every ball in $\mathcal{J}_{q}$ contains $J$ with arbitrarily big $\left|J_{x y}\right|$. Then the interaction randomness is realized as the triple $\left(\mathcal{J}_{q}, \mathcal{B}\left(\mathcal{J}_{q}\right), \nu\right)$, where $\nu$ is a general complete probability measure (need not be product, etc). For every finite $\Delta \subset \mathbb{Z}^{d}$, by means of the potentials (1.3) we introduce the local conditional Gibbs measure $\pi_{\Delta}(\cdot \mid J, \xi), J \in \mathcal{J}_{q}$ and $\xi \in \mathcal{S}_{p}$, which then allows us to define the set of tempered Gibbs measures $\mathcal{G}_{p}(J)$ consisting of those $\mu \in \mathcal{P}\left(\mathbb{R}^{\mathbb{Z}^{d}}\right)$ which solve the DLR equation and are such that $\mu\left(\mathcal{S}_{p}\right)=1$. We claim that:

(a) for every $J \in \mathcal{J}_{q}$, the set $\mathcal{G}_{p}(J)$ is non-void and weakly compact, and that each $\mu \in \mathcal{G}_{p}(J)$ obeys an integrability estimate, the same for all such $\mu$ (Theorem 3.1);

(b) the map $\mathcal{J}_{q} \ni J \mapsto \mathcal{G}_{p}(J)$ is measurable, as a set-valued map, and hence there exist measurable selections $\mathcal{J}_{q} \ni J \mapsto \mu(J) \in \mathcal{G}_{p}(J)$ (Theorem 3.4 $)$.

The key element of the proof of (a) is an integrability estimate for the measures $\pi_{\Delta}(\cdot \mid J, \xi)$ which implies the existence of accumulation points of the family $\left\{\pi_{\Delta}(\cdot \mid J, \xi)\right\}_{\Delta \subset \mathbb{Z}^{d}}$, that are elements of $\mathcal{G}_{p}(J)$. Then, the corresponding estimate for $\mu \in \mathcal{G}_{p}(J)$, which holds uniformly for all such $\mu$ and all $\|J\|_{q} \leqslant R, R>0$, are obtained therefrom. This allows us to prove that the map $\mathcal{J}_{q} \ni J \mapsto \mathcal{G}_{p}(J)$ is upper semi-continuous, which extends the result obtained (for bounded interactions) in item (d) of Theorem 4.23 in [6], page 72. By Theorem 8.1.4 of [2], page 310, the mentioned upper semicontinuity implies the measurability of $\mathcal{J}_{q} \ni J \mapsto \mathcal{G}_{p}(J)$, which in turn yields the existence of

\footnotetext{
${ }^{1}$ The a.s. uniqueness of Gibbs measures of disordered spin systems, as well as the problem of phase transitions, are highly nontrivial, see the discussion and the corresponding references in section 6.3 in [1].
} 
measurable selections, see Theorem 8.1.3 in [2]. In Corollary 3.2, we also establish the existence of the averages $\mathbb{E}_{\nu} \Phi\left(\mathbb{E}_{\mu(J)} F\right)$ for appropriate functions $F$ and $\Phi$. Note that the constants in (3.3) are explicitly expressed in terms of the model parameters.

As is commonly accepted, see chapter 7 in [6] , the extreme elements of $\mathcal{G}_{p}(J)$ correspond to thermodynamic phases of the physical system modeled by the family $\left\{\pi_{\Delta}(\cdot \mid J, \xi)\right\}_{\Delta \subset \mathbb{Z}^{d}}$. These elements are contained in the set of limiting Gibbs measures (Minlos states), see Corollary 7.30 on page 135 in [6] , which are exactly the accumulation points of $\left\{\pi_{\Delta}(\cdot \mid J, \xi)\right\}_{\Delta \subset \mathbb{Z}^{d}}$. The physical meaning of such limiting Gibbs measures is that they approximate Gibbs measures of large finite systems, c.f. the corresponding discussion in 7]. The random Gibbs measures obtained in (b) as measurable selections need not be limiting Gibbs measures - thus, the result of Theorem 3.4 has rather got a theoretical value from the point of view of physics. The characteristic feature of the spin models with random interactions is the so-called chaotic dependence of the measures $\pi_{\Delta}(\cdot \mid J, \xi)$ on $\Delta$, see [8] and the references cited therein. This means that the limits of the sequences $\left\{\pi_{\Delta_{n}}(\cdot \mid J, \xi)\right\}_{n \in \mathbb{N}}$ need not be measurable (with respect to $J$ ) and hence cannot serve as limiting random Gibbs measures. With the help of the Komlós theorem [9, 10] in Theorem 3.6 we obtain that,

(c) for every $\xi \in \mathcal{S}_{p}$, there exists a random Gibbs measure $\mu^{\xi}(J)$ and an exhausting sequence $\mathcal{D}=\left\{\Delta_{n}\right\}_{n \in \mathbb{N}}$ such that $\mu^{\xi}(J)$ is the $\nu$-a.s. weak limit of the sequence of 'empirical distributions'

$$
\frac{1}{N} \sum_{n=1}^{N} \pi_{\Delta_{n}}(\cdot \mid J, \xi), \quad N \in \mathbb{N}
$$

Under rather general assumptions, each Gibbs measure has an extreme decomposition, see Theorem 7.26 in [6], page 133. Thus, every measurable selection can be written in the form

$$
\mu(J)=\int_{\mathcal{G}_{p}^{\text {ex }}(J)} \mu \mathfrak{w}(J)(\mathrm{d} \mu) .
$$

Here $\mathcal{G}_{p}^{\text {ex }}(J)$ is the extreme boundary of $\mathcal{G}_{p}(J)$ and $\mathfrak{w}(J)$ is a weight, uniquely determined by $\mu(J)$. This decomposition holds for all $J \in \mathcal{J}_{q}$, however, the weights $\mathfrak{w}(J)$ need not be $J$-measurable. Suppose now that a representation holds, which is similar to (1.5) with a measurable weight and the integral taken over the whole set $\mathcal{G}_{p}(J)$. Then it yields a random Gibbs measure and the corresponding weight is called an Aizenman-Wehr metastate [11], see also page 103 in [1] and Definition 2.4 below. In Theorem 3.7 we show that

(d) for every $\xi \in \mathcal{S}_{p}$, there exists an Aizenman-Wehr metastate $\mathfrak{m}^{\xi}(J)$ and an exhausting sequence $\mathcal{D}=\left\{\Delta_{n}\right\}_{n \in \mathbb{N}}$ such that $\mathfrak{m}^{\xi}(J)$ is the $\nu$-a.s. weak limit of the sequence of empirical metastates $\left\{N^{-1} \sum_{n=1}^{N} \delta_{\pi_{\Delta_{n}}(\cdot \mid J, \xi)}\right\}_{N \in \mathbb{N}}$.

The thermodynamic pressure, or the free energy density, is an important characteristic which one obtains in the thermodynamic limit, see. e.g. the discussion in [1], p. 24-28. For non-random (translation invariant) systems, the pressure exists and is independent of the way the limit has been taken, see sections 2 and 3 in [3] or Theorem 3.10 in [12]. In Theorem 3.8, we show that under an additional condition on the measure $\nu$ the pressure can be obtained as the almost sure limit of the local pressures, 'averaged' over $\left\{\Delta_{n}\right\}$ similarly as in (1.4). In Theorem 3.9. we assume that $\nu$ is a product measure with the zero first moment and prove that all the sequences of local pressures averaged over the disorder have one and the same thermodynamic limit - the quenched pressure. In proving Theorems 3.8 and 3.9 we employ a version of the first GKS inequality, known for such models with $J_{x y} \geqslant 0$, which we obtain here by extending the approach of [13, 14] to the case of unbounded interactions. 


\section{Setup}

\subsection{General setting}

In constructing Gibbs random fields, we follow the standard scheme [6]. Our Gibbs fields will live on the set $\mathbf{X}=\mathbb{Z}^{d}, d \in \mathbb{N}$, equipped with the adjacency relation $x \sim y$ defined by the condition $|x-y|=1$. By $\mathrm{E}$ we denote the set of edges of the corresponding graph. We also use the shorthand

$$
\sum_{x}=\sum_{x \in \mathbb{Z}^{d}}, \quad \sup _{x}=\sup _{x \in \mathbb{Z}^{d}}, \quad \sum_{y \sim x}=\sum_{y \in \mathbb{Z}^{d}: y \sim x} .
$$

The set $\mathbb{R}^{\mathbb{Z}^{d}}$ is equipped with the product topology, which turns it into a Polish space - a separable and completely metrizable topological space. Let $C_{\mathrm{b}}\left(\mathbb{R}^{\mathbb{Z}^{d}}\right)$ be the Banach space of bounded continuous functions $f: \mathbb{R}^{\mathbb{Z}^{d}} \rightarrow \mathbb{R}$ with the norm

$$
\|f\|_{\infty}=\sup _{\sigma \in \mathbb{R}^{\mathbb{Z}^{d}}}|f(\sigma)|
$$

By means of $C_{\mathrm{b}}\left(\mathbb{R}^{\mathbb{Z}^{d}}\right)$ we define the weak topology on the set of all probability measures $\mathcal{P}\left(\mathbb{R}^{\mathbb{Z}^{d}}\right)$, which turns it into a Polish space, see e.g. page 39 in [15].

For any $\Delta \subset \mathbb{Z}^{d}$, we let $\Delta^{\mathrm{c}} \stackrel{\text { def }}{=} \mathbb{Z}^{d} \backslash \Delta$; by writing $\Delta \Subset \mathrm{X}$ we mean that $0<|\Delta|<\infty$. A sequence $\mathcal{D}=\left\{\Delta_{n}\right\}_{n \in \mathbb{N}}$, such that $\Delta_{n} \Subset \mathbb{Z}^{d}$ for all $n \in \mathbb{N}$, is said to be cofinal if it is: (a) ordered by inclusion; (b) exhausting, i.e. such that each $x \in \mathbb{Z}^{d}$ belongs to a certain $\Delta_{n}$. For $\Delta \subset \mathbb{Z}^{d}$, by $\mathcal{B}_{\Delta}$ we denote the $\sigma$-sub-field of $\mathcal{B}\left(\mathbb{R}^{\mathbb{Z}^{d}}\right)$ generated by $(\sigma(x))_{x \in \Delta}$. For $\Delta \Subset \mathbb{Z}^{d}$, a probability kernel $\pi_{\Delta}(\cdot \mid \cdot)$ is a function on $\left(\mathcal{B}\left(\mathbb{R}^{\mathbb{Z}^{d}}\right), \mathbb{R}^{\mathbb{Z}^{d}}\right)$ such that for any $\xi \in \mathbb{R}^{\mathbb{Z}^{d}}, \pi_{\Delta}(\cdot \mid \xi)$ is in $\mathcal{P}\left(\mathbb{R}^{\mathbb{Z}^{d}}\right)$, and for any $A \in \mathcal{B}\left(\mathbb{R}^{\mathbb{Z}^{d}}\right), \pi_{\Delta}(A \mid \cdot)$ is $\mathcal{B}_{\Delta^{\mathrm{c}}}$-measurable. Such a kernel is said to be proper if $\pi_{\Delta}(A \mid \cdot)=\mathbb{I}_{A}(\cdot)$ for any $A \in \mathcal{B}_{\Delta^{\mathrm{c}}}$. Here $\mathbb{I}_{A}(\xi)=1$ if $\xi \in A$, and $\mathbb{I}_{A}(\xi)=0$ otherwise. Given a family $\left\{\pi_{\Delta}\right\}_{\Delta \Subset \mathbb{Z}^{d}}$, suppose that there exists $\mu \in \mathcal{P}\left(\mathbb{R}^{\mathbb{Z}^{d}}\right)$ such that

$$
\mu\left(A \mid \mathcal{B}_{\Delta^{\mathrm{c}}}\right)=\pi_{\Delta}(A \mid \cdot),
$$

which holds $\mu$-almost surely for all $A \in \mathcal{B}\left(\mathbb{R}^{\mathbb{Z}^{d}}\right)$ and $\Delta \Subset \mathbb{Z}^{d}$. Then this measure $\mu$ is said to be specified by the family $\left\{\pi_{\Delta}\right\}_{\Delta \Subset \mathbb{Z}^{d}}$. In this case, all the kernels $\pi_{\Delta}$ are $\mu$-almost surely proper, and their family is $\mu$-almost surely consistent. The latter means that for $\mu$-almost all $\xi$ and all $A \in \mathcal{B}\left(\mathbb{R}^{\mathbb{Z}^{d}}\right)$

$$
\int_{\mathbb{R}^{\mathbb{Z}^{d}}} \pi_{\Lambda}(A \mid \eta) \pi_{\Delta}(\mathrm{d} \eta \mid \xi)=\pi_{\Delta}(A \mid \xi)
$$

which holds for any pair of subsets such that $\Lambda \subset \Delta$. It should be pointed out that (2.1) is equivalent to

$$
\int_{\mathbb{R}^{\mathbb{Z}^{d}}} \pi_{\Delta}(A \mid \xi) \mu(\mathrm{d} \xi)=\mu(A),
$$

which holds for all $A \in \mathcal{B}\left(\mathbb{R}^{\mathbb{Z}^{d}}\right)$ and $\Delta \Subset \mathbb{Z}^{d}$. The condition (2.3) is called the Dobrushin-LanfordRuelle $(D L R)$ equation. It is equivalent to

$$
\int_{\mathbb{R}^{\mathbb{Z}^{d}}} \pi_{\Delta}(f \mid \xi) \mu(\mathrm{d} \xi)=\mu(f)
$$

for all $f \in C_{\mathrm{b}}\left(\mathbb{R}^{\mathbb{Z}^{d}}\right)$ and all $\Delta \Subset \mathbb{Z}^{d}$. Here we use the notation

$$
\mu(f)=\int_{\mathbb{R}^{\mathbb{Z}^{d}}} f(\sigma) \mu(\mathrm{d} \sigma)
$$




\subsection{The Gibbs fields}

The Gibbs fields we are going to construct are specified by the kernels obtained as perturbations of the products of single-spin measures by the factors (1.1) with the functions $W_{x y}$ as in (1.3). For $\Delta \Subset \mathbb{Z}^{d}$ and $\xi \in \mathbb{R}^{\mathbb{Z}^{d}}$, we set

$$
-H_{\Delta}\left(\sigma_{\Delta} \mid J, \xi\right)=\sum_{\langle x, y\rangle \in \mathrm{E}_{\Delta}} J_{x y} \sigma(x) \sigma(y)+\sum_{x \in \Delta,} \sum_{y \in \Delta^{\mathrm{c}}, x \sim y} J_{x y} \sigma(x) \xi(y),
$$

where $\mathrm{E}_{\Delta}$ consists of the edges with both endpoints in $\Delta$. In the mentioned terminology, $H_{\Delta}\left(\sigma_{\Delta} \mid J, \xi\right)$ is the energy of interaction of the spins located in $\Delta$ with each other and with the fixed spins outside $\Delta$. For a family $\chi=\left(\chi_{x}\right)_{x \in \mathbb{Z}^{d}}, \chi_{x} \in \mathcal{P}(\mathbb{R})$, we put

$$
\chi_{\Delta}\left(\mathrm{d} \sigma_{\Delta}\right)=\prod_{x \in \Delta} \chi_{x}(\mathrm{~d} \sigma(x)), \quad \sigma_{\Delta}=(\sigma(x))_{x \in \Delta}
$$

which is an element of $\mathcal{P}\left(\mathbb{R}^{|\Delta|}\right)$. Thereafter, for $A \in \mathcal{B}\left(\mathbb{R}^{\mathbb{Z}^{d}}\right)$, we define

$$
\pi_{\Delta}(A \mid J, \xi)=\frac{1}{Z_{\Delta}(J, \xi)} \int_{\mathbb{R}^{|\Delta|}} \mathbb{I}_{A}\left(\sigma_{\Delta} \times \xi_{\Delta^{\mathrm{c}}}\right) \exp \left[-H_{\Delta}\left(\sigma_{\Delta} \mid J, \xi\right)\right] \chi_{\Delta}\left(\mathrm{d} \sigma_{\Delta}\right) .
$$

Here $Z_{\Delta}(J, \xi)$ is a normalizing factor, that is,

$$
Z_{\Delta}(J, \xi)=\int_{\mathbb{R}^{|\Delta|}} \exp \left[-H_{\Delta}\left(\sigma_{\Delta} \mid J, \xi\right)\right] \chi_{\Delta}\left(\mathrm{d} \sigma_{\Delta}\right)
$$

and the juxtaposition stands for the element of $\mathbb{R}^{\mathbb{Z}^{d}}$ such that

$$
\left(\sigma_{\Delta} \times \xi_{\Delta^{\mathrm{c}}}\right)(x)=\sigma(x), \quad \text { for } x \in \Delta ; \quad\left(\sigma_{\Delta} \times \xi_{\Delta^{\mathrm{c}}}\right)(x)=\xi(x), \quad \text { for } x \in \Delta^{\mathrm{c}}
$$

The family $\left\{\pi_{\Delta}\right\}_{\Delta \Subset \mathbb{Z}^{d}}$ is clearly consistent. It is the local Gibbs specification for our model.

As is typical of Gibbs measures of models with unbounded spins, the description of the properties possessed by all such measures is rather unrealistic. Usually, the study is restricted to those measures which have a prescribed support property. Such measures are called tempered. To define the mentioned property we use a weight function $w: \mathbb{Z}^{d} \rightarrow(0,1]$, which by definition has the following properties:

$$
\begin{aligned}
& \quad|w| \stackrel{\text { def }}{=} \sum_{x} w(x)<\infty, \\
& \text { (b) } \quad \exists w_{0}>0 \quad w(x) \leqslant w_{0} w(y), \quad \text { for all } x \sim y .
\end{aligned}
$$

Note that $w_{0} \geqslant 1$, otherwise one would get $w(x) \equiv 0$. A typical example can be

$$
w(x)=\exp (-\alpha|x|), \quad \alpha>0 .
$$

For $w$ obeying (2.10) and (2.11) and for a $p \geqslant 1$, we set

$$
\|\sigma\|_{p}=\left(\sum_{x}|\sigma(x)|^{p} w(x)\right)^{1 / p}
$$

and

$$
\mathcal{S}_{p}=L^{p}\left(\mathbb{Z}^{d}, w\right)=\left\{\sigma \in \mathbb{R}^{\mathbb{Z}^{d}}:\|\sigma\|_{p}<\infty\right\}
$$


This will be the space of tempered spin configurations. Next, for $q \geqslant 1$, we introduce the space of tempered interaction intensities

$$
\begin{aligned}
& \|J\|_{q}=\left(\sum_{\langle x, y\rangle \in \mathrm{E}}\left|J_{x y}\right|^{q}[w(x)+w(y)]\right)^{1 / q}, \\
& \mathcal{J}_{q}=L^{q}(\mathrm{E}, w)=\left\{J \in \mathbb{R}^{\mathrm{E}}:\|J\|_{q}<\infty\right\} .
\end{aligned}
$$

Clearly, $L^{p}\left(\mathbb{Z}^{d}, w\right)$ and $L^{q}(\mathrm{E}, w)$ are measurable subsets of the Polish spaces $\mathbb{R}^{\mathbb{Z}^{d}}$ and $\mathbb{R}^{\mathrm{E}}$, respectively. We equip these sets with the corresponding norm topologies, which turns them into separable Banach spaces. It can easily be shown (see also the Kuratowski theorem, page 15 in [15]), that

$$
\mathcal{B}\left(\mathcal{S}_{p}\right)=\left\{\mathcal{S}_{p} \cap A: A \in \mathcal{B}\left(\mathbb{R}^{\mathbb{Z}^{d}}\right)\right\}
$$

Thus, one can consider the set

$$
\mathcal{P}_{\text {temp }}=\left\{\mu \in \mathcal{P}\left(\mathbb{R}^{\mathbb{Z}^{d}}\right): \mu\left(\mathcal{S}_{p}\right)=1\right\}
$$

The elements of $\mathcal{P}_{\text {temp }}$ are called tempered measures. Now we impose conditions on the family of single-spin measures $\chi=\left(\chi_{x}\right)_{x \in \mathbb{Z}^{d}}$. For $\lambda>0$ and $q>1$, we set

$$
\begin{aligned}
& \sup _{x} \int_{\mathbb{R}} \exp \left(\lambda|u|^{2 q /(q-1)}\right) \chi_{x}(\mathrm{~d} u)=C_{+}(\lambda), \\
& \inf _{x} \int_{\mathbb{R}} \exp \left(-\lambda|u|^{2 q /(q-1)}\right) \chi_{x}(\mathrm{~d} u)=C_{-}(\lambda) .
\end{aligned}
$$

And then

$$
\mathcal{K}_{q} \stackrel{\text { def }}{=}\left\{\chi=\left(\chi_{x}\right)_{x \in \mathbb{Z}^{d}}: \forall \lambda>0 \quad C_{+}(\lambda)<\infty, C_{-}(\lambda)>0\right\}
$$

As an example of $\chi \in \mathcal{K}_{q}$ one can take the copies of the measure $\chi_{0}(d u) \sim \exp (-V(u)) d u$, where $V$ is an even semi-bounded polynomial of $\operatorname{deg} V>2 q /(q-1)$, c.f. [3], 5]. This corresponds to the physical model called an anharmonic crystal where the spins are the displacements of the oscillators from their equilibrium positions.

In the sequel, we shall always choose $J$ in $\mathcal{J}_{q}$ and $\chi$ in $\mathcal{K}_{q}$ with one and the same $q>1$. We also assume that this $q$ and $p$ in (2.14) and (2.17) satisfy

$$
p=\frac{2 q}{q-1}
$$

i.e. $p>2$. As our main concern is the dependence on $J$, the dependence on $\chi$ will always be suppressed from the notations.

Definition 2.1 Given $J=\left(J_{x y}\right)_{\langle x, y\rangle \in \mathrm{E}} \in \mathcal{J}_{q}$ and $p$ as in (2.20), by $\mathcal{G}_{p}(J)$ we denote the set of all $\mu \in \mathcal{P}_{\text {temp }}$ which solve the DLR equation (2.3) with the kernels defined in (2.6) and (2.8). The elements of $\mathcal{G}_{p}(J)$ are called (tempered) Gibbs measures.

We recall that a probability space $(\Omega, \mathcal{O}, P)$ is said to be complete if for every $A$ such that $P(A)=0$, each subset of $A$ is in $\mathcal{O}$. We also recall that $\mathcal{J}_{q}$ is a separable Banach space.

Definition 2.2 By the lattice model with unbounded spins and unbounded random interactions we mean the pair

$$
\left(\mathcal{J}_{q}, \mathcal{B}\left(\mathcal{J}_{q}\right), \nu\right) \text { and }\left\{\pi_{\Delta}(\cdot \mid J, \xi): \Delta \Subset \mathbb{Z}^{d}, J \in \mathcal{J}_{q}, \xi \in \mathcal{S}_{p}\right\}
$$

where the probability space is complete and the kernels $\pi_{\Delta}$ are defined in (2.6) and (2.8). 
Definition 2.3 A $\mathcal{B}\left(\mathcal{J}_{q}\right) / \mathcal{B}\left(\mathcal{P}\left(\mathbb{R}^{\mathbb{Z}^{d}}\right)\right)$-measurable map $\mathcal{J}_{q} \ni J \mapsto \mu(J) \in \mathcal{P}\left(\mathbb{R}^{\mathbb{Z}^{d}}\right)$ is said to be a random Gibbs measure if $\mu(J) \in \mathcal{G}_{p}(J)$ for $\nu$-almost all $J \in \mathcal{J}_{q}$.

Note that when we speak about a Gibbs measure $\mu$ we mean merely an element of a given $\mathcal{G}_{p}(J)$. However, a random Gibbs measure $\mu(J)$ will stand for a measure-valued function of $J \in \mathcal{J}_{q}$.

Let $\mathfrak{P}$ denote the space of all probability measures on $\left(\mathcal{P}\left(\mathbb{R}^{\mathbb{Z}^{d}}\right), \mathcal{B}\left(\mathcal{P}\left(\mathbb{R}^{\mathbb{Z}^{d}}\right)\right)\right)$. We equip it with the weak topology and thereby with the Borel $\sigma$-field $\mathfrak{B}$. For every $f \in C_{\mathrm{b}}\left(\mathbb{R}^{\mathbb{Z}^{d}}\right)$, the evaluation map $\mathcal{P}\left(\mathbb{R}^{\mathbb{Z}^{d}}\right) \ni \mu \mapsto \mu(f)$ is continuous and bounded.

Definition 2.4 A B $\left(\mathcal{J}_{q}\right) / \mathfrak{B}$-measurable map $\mathcal{J}_{q} \ni J \mapsto \mathfrak{m}(J) \in \mathfrak{P}$ is said to be an Aizenman-Wehr metastate if

(a) $\mathfrak{m}(J)\left(\mathcal{G}_{p}(J)\right)=1$ for $\nu$-almost all $J \in \mathcal{J}_{q}$;

(b) the map

$$
\mathcal{J}_{q} \ni J \mapsto \int_{\mathcal{P}\left(\mathbb{R}^{\mathbb{Z}^{d}}\right)} \mu \mathfrak{m}(J)(\mathrm{d} \mu) \in \mathcal{P}\left(\mathbb{R}^{\mathbb{Z}^{d}}\right)
$$

is a random Gibbs measure.

Note that the integral in (2.21) is understood in terms of the pairing with $f \in C_{\mathrm{b}}\left(\mathbb{R}^{\mathbb{Z}^{d}}\right)$.

\section{The results}

\subsection{Theorems}

For $R>0$, we set $B_{q}(R)=\left\{J \in \mathcal{J}_{q}:\|J\|_{q} \leqslant R\right\}$. From (2.15) it follows that

$$
\sup _{J \in B_{q}(R)} \sup _{\langle x, y\rangle \in \mathrm{E}}\left|J_{x y}\right|=+\infty
$$

for any $R>0$. Recall that the set of tempered measures $\mathcal{P}_{\text {temp }}$ was defined in (2.17). In the sequel, when we discuss topological properties of $\mathcal{G}_{p}(J)$ we always mean the topology induced by the weak topology of the space $\mathcal{P}\left(\mathbb{R}^{\mathbb{Z}^{d}}\right)$, defined by means of $C_{\mathrm{b}}\left(\mathbb{R}^{d}\right)$.

Theorem 3.1 For every $J \in \mathcal{J}_{q}, q>1$, the set $\mathcal{G}_{p}(J)$ ( $p$ as in (2.20) $)$ is non-void and compact. For any $\lambda>0$, there exist positive constants $\Upsilon_{i}(\lambda), i=1,2$, such that for every $\mu \in \mathcal{G}_{p}(J)$, the following estimate holds

$$
\int_{\mathbb{R}^{\mathbb{Z}^{d}}} \exp \left(\lambda\|\sigma\|_{p}^{p}\right) \mu(\mathrm{d} \sigma) \leqslant \exp \left(\Upsilon_{1}(\lambda)+\Upsilon_{2}(\lambda)\|J\|_{q}^{q}\right) .
$$

By Jensen's inequality, one readily gets from (3.2) the following.

Corollary 3.2 There exist positive constants $A$ and $B$ such that for any random Gibbs measure $\mu(J)$, the following estimate

$$
\int_{\mathcal{J}_{q}} \Phi\left(\int_{\mathbb{R}^{\mathbb{Z}^{d}}}\|\sigma\|_{p}^{p} \mu(J)(\mathrm{d} \sigma)\right) \nu(\mathrm{d} J) \leqslant \int_{\mathcal{J}_{q}} \Phi\left(A+B\|J\|_{q}^{q}\right) \nu(\mathrm{d} J)
$$

holds for any increasing function $\Phi: \mathbb{R}_{+} \rightarrow \mathbb{R}_{+}$.

Random Gibbs measures can be obtained as measurable selections. 
Definition 3.3 A measurable map $\mathcal{J}_{q} \ni J \mapsto \mu(J) \in \mathcal{P}\left(\mathbb{R}^{\mathbb{Z}^{d}}\right)$ such that

$$
\forall J \in \mathcal{J}_{q}: \quad \mu(J) \in \mathcal{G}_{p}(J)
$$

is called a measurable selection of the set-valued map $\mathcal{J}_{q} \ni J \mapsto \mathcal{G}_{p}(J) \subset \mathcal{P}\left(\mathbb{R}^{\mathbb{Z}^{d}}\right)$.

Theorem 3.4 Let $p$ and $q$ be as in Theorem 3.1 and the probability space $\left(\mathcal{J}_{q}, \mathcal{B}\left(\mathcal{J}_{q}\right), \nu\right)$ be as in Definition 2.2. Then the map $\mathcal{J}_{q} \ni J \mapsto \mathcal{G}_{p}(J)$ has measurable selections.

It turns out that measurable selections constitute quite a big subset of the set of Gibbs measures, c.f. item (vi) of Theorem 8.1.4 in [2], page 310 .

Remark 3.5 There exists an at most countable family $\left\{\mu_{n}\right\}_{n \in \mathbb{N}}$ of measurable selections mentioned in Theorem 3.4 such that, for every $J \in \mathcal{J}_{q}$, the set $\left\{\mu_{n}(J)\right\}_{n \in \mathbb{N}} \subset \mathcal{G}_{p}(J)$ is dense in $\mathcal{G}_{p}(J)$. Thus, $\mathcal{G}_{p}(J)$ is a singleton for $\nu$-almost all $J$ if there is only one measurable selection.

As was already mentioned in Introduction, only limiting Gibbs measures can serve as the approximations of the Gibbs measures of large finite systems, see [7]. In the next theorem, we obtain random Gibbs measures as weak limits of the averaged kernels $\pi_{\mathcal{D}, N}$. For a cofinal sequence $\mathcal{D}=\left\{\Delta_{n}\right\}_{n \in \mathbb{N}}$ and $N \in \mathbb{N}$, we set

$$
\pi_{\mathcal{D}, N}(\cdot \mid J, \xi)=\frac{1}{N} \sum_{n=1}^{N} \pi_{\Delta_{n}}(\cdot \mid J, \xi) .
$$

Theorem 3.6 For every $\xi \in \mathcal{S}_{p}$, there exists a random Gibbs measure $\mu^{\xi}$ and a cofinal sequence $\mathcal{D}$ such that, in the topology of $\mathcal{P}\left(\mathbb{R}^{\mathbb{Z}^{d}}\right)$, one has $\mu^{\xi}(J)=\lim _{N \rightarrow+\infty} \pi_{\mathcal{D}, N}(\cdot \mid J, \xi)$ for $\nu$-almost all $J \in \mathcal{J}_{q}$.

The fact that we use the sequences of averaged kernels to approximate the finite volume Gibbs measures rather than the sequences of kernels themselves can be explained by the chaotic dependence of the kernels $\pi_{\Delta}(\cdot \mid J, \xi)$ on $\Delta$, which is smoothed up in (3.4).

For $\Delta \Subset \mathbb{Z}^{d}$, let $\mathfrak{d}_{\Delta}^{\xi}(J)$ denote the $\delta$-measure centered at $\pi_{\Delta}(\cdot \mid J, \xi)$, that is $\mathfrak{d}_{\Delta}^{\xi}(J)(A)=$ $\mathbb{I}_{A}\left(\pi_{\Delta}(\cdot \mid J, \xi)\right)$ for all $A \in \mathfrak{B}$. Then for a cofinal sequence $\mathcal{D}$ and $N \in \mathbb{N}$, we set, c.f. (3.4),

$$
\mathfrak{d}_{\mathcal{D}, N}^{\xi}(J)=\frac{1}{N} \sum_{n=1}^{N} \mathfrak{d}_{\Delta_{n}}^{\xi}(J),
$$

which is the Newman-Stein empirical metastate, see equation (B19) in page 77 in [16] or equation (A18) in page 281 in [8]. Recall that the Aizenman-Wehr metastates were introduced in Definition 2.4.

Theorem 3.7 For every $\xi \in \mathcal{S}_{p}$, there exists an Aizenman-Wehr metastate $\mathfrak{m}^{\xi}$ and a cofinal sequence $\mathcal{D}$ such that, in the topology of $\mathfrak{P}, \mathfrak{m}^{\xi}(J)=\lim _{N \rightarrow+\infty} \mathfrak{d}_{\mathcal{D}, N}^{\xi}(J)$ for $\nu$-almost all $J \in \mathcal{J}_{q}$.

For $\Delta \Subset \mathbb{Z}^{d}, J \in \mathcal{J}_{q}$, and $\xi \in \mathcal{S}_{p}$, the (local) pressure in $\Delta$ is

$$
p_{\Delta}(J, \xi)=\frac{1}{|\Delta|} \log Z_{\Delta}(J, \xi),
$$

where $Z_{\Delta}(J, \xi)$ is the same as in (2.9). Like in (3.4), for a cofinal sequence $\mathcal{D}=\left\{\Delta_{n}\right\}_{n \in \mathbb{N}}$ and $N \in \mathbb{N}$, we consider

$$
p_{\mathcal{D}, N}(J, \xi)=\frac{1}{N} \sum_{n=1}^{N} p_{\Delta_{n}}(J, \xi) .
$$


Let now $\mu$ be a random Gibbs measure, see Definition 2.3. Then

$$
\begin{aligned}
\bar{p}_{\Delta}^{\mu}(J) & =\int_{\mathbb{R}^{\mathbb{Z}^{d}}} p_{\Delta}(J, \xi) \mu(J)(\mathrm{d} \xi), \\
\bar{p}_{\mathcal{D}, N}^{\mu}(J) & =\int_{\mathbb{R}^{\mathbb{Z}^{d}}} p_{\mathcal{D}, N}(J, \xi) \mu(J)(\mathrm{d} \xi),
\end{aligned}
$$

are measurable functions of $J \in \mathcal{J}_{q}$, and

$$
\vartheta(\mathrm{d} \sigma, \mathrm{d} J)=\mu(J)(\mathrm{d} \sigma) \nu(\mathrm{d} J)
$$

is a probability measure on the product space $\mathbb{R}^{\mathbb{Z}^{d}} \times \mathcal{J}_{q}$.

Theorem 3.8 Suppose that $\nu$ has the property

$$
\sup _{\langle x, y\rangle \in \mathrm{E}} \int_{\mathcal{J}_{q}}\left|J_{x y}\right|^{q} \nu(\mathrm{d} J)=a_{\nu}<+\infty .
$$

Then, for any random Gibbs measure $\mu$, there exists a cofinal sequence $\mathcal{D}$ such that the sequence $\left\{\bar{p}_{\mathcal{D}, N}^{\mu}(J)\right\}_{N \in \mathbb{N}}$ converges, for $\nu$-almost all $J \in \mathcal{J}_{q}$, to a certain $p^{\mu} \in L^{1}\left(\mathcal{J}_{q}, \nu\right)$. Furthermore, for $\nu$ obeying (3.10), let $\vartheta$ be as in (3.9). Then there exists a cofinal sequence $\mathcal{D}$ such that the sequence $\left\{p_{\mathcal{D}, N}(J, \xi)\right\}_{N \in \mathbb{N}}$ converges, for $\vartheta$-almost all $(\xi, J) \in \mathbb{R}^{\mathbb{Z}^{d}} \times \mathcal{J}_{q}$, to a certain $p \in L^{1}\left(\mathbb{R}^{\mathbb{Z}^{d}} \times \mathcal{J}_{q}, \vartheta\right)$.

Imposing an additional condition on the measure $\nu$ we can strengthen the above result as follows. A cofinal sequence $\mathcal{D}=\left\{\Delta_{n}\right\}_{n \in \mathbb{N}}$ is called a van Hove sequence if

$$
\inf _{n \in \mathbb{N}} \frac{\left|\partial \Delta_{n}\right|}{\left|\Delta_{n}\right|}=\lim _{n \rightarrow+\infty} \frac{\left|\partial \Delta_{n}\right|}{\left|\Delta_{n}\right|}=0
$$

see e.g. page 193 in [17]. Here $\partial \Delta=\left\{y \in \Delta^{\mathrm{c}}: \exists x \in \Delta x \sim y\right\}$.

Theorem 3.9 In addition to (3.10), assume that $\nu$ is a product measure such that

$$
\int_{\mathcal{J}_{q}} J_{x y} \nu(\mathrm{d} J)=0
$$

for all $\langle x, y\rangle \in \mathrm{E}$. Then, for any cofinal sequence $\mathcal{D}=\left\{\Delta_{n}\right\}_{n \in \mathbb{N}}$, there exists the quenched pressure

$$
p^{\text {quen }}=\lim _{n \rightarrow+\infty} \int_{\mathcal{J}_{q}} p_{\Delta_{n}}(J, 0) \nu(\mathrm{d} J)=\sup _{\Delta \Subset \mathbb{Z}^{d}} \int_{\mathcal{J}_{q}} p_{\Delta}(J, 0) \nu(\mathrm{d} J),
$$

which thereby is independent of $\mathcal{D}$. Furthermore, for any random Gibbs measure $\mu$ and any van Hove sequence $\mathcal{D}=\left\{\Delta_{n}\right\}_{n \in \mathbb{N}}$, we have that

$$
p^{\text {quen }}=\lim _{n \rightarrow+\infty} \int_{\mathcal{J}_{q}} \bar{p}_{\Delta_{n}}^{\mu}(J) \nu(\mathrm{d} J)
$$

\subsection{Comments}

As was already mentioned, the model with the interaction as in (2.6), and with the singlespin measures $\chi_{0}(\mathrm{~d} u) \sim \exp (-V(u)) \mathrm{d} u$, describes an anharmonic crystal with unbounded random interactions. On the other hand, this is an irregular model, which has not been studied yet and it seems a challenging task to develop its mathematical theory. We plan to include a random external 
field into consideration in a separate work, c.f. 11]. All the results presented above can be readily extended to any bounded degree graph, and, after some modifications, to unbounded degree graphs of a certain kind [18]. They can also be extended to more general pair interaction potentials $W_{x y}$, c.f. (1.3). If every single-spin measure $\chi_{x}$ is supported on a bounded $[a, b]$, then all the results formulated above hold true with any $q$ and $p=2 q /(q-1)$, including $q=1$ and $p=\infty$. In this case, we deal with regular random interaction $\$$. An important particular model of this kind is the Edwards-Anderson spin glass, see section 2 in [8]. In this model, the spins take values \pm 1 with equal probabilities and the interaction intensities $J_{x y}$ are symmetric, typically Gaussian, i.i.d.. Note that such a model meets the conditions of Theorem 3.9

More specific remarks to the above results are as follows:

- Theorem 3.1. The main point of this theorem is the lack of uniform boundedness of the intensities $J_{x y}$, c.f. (3.1). Clearly, the growth of $J_{x y}$ should be controlled in one or another way. We do this by imposing the temperedness condition $\|J\|_{q}<\infty$, which appears in the right-hand side of (3.2) and in similar estimates. The same results can be obtained for the Euclidean Gibbs measures which describe equilibrium thermodynamic states of lattice systems of interacting quantum anharmonic oscillators with random interactions. In this case, our Theorem 3.1 would be an extension of Theorems 3.1 and 3.2 of [12] and of Theorems 3.3.1 and 3.3.6, p. 214-216 in [17]. For the Euclidean Gibbs measures, the single-spin spaces $S_{x}$ are the copies of the space of periodic continuous functions $\sigma_{x}:[0, \beta] \rightarrow \mathbb{R}$, where $\beta>0$ is the inverse temperature. In view of this, one needs to apply more sophisticated methods of the path integral approach [17].

- Theorem 3.4. If $J$ is random and fixed, the set $\mathcal{G}_{p}(J)$ describes the equilibrium thermodynamic states of the spin system with quenched disorder. In order to average over the disorder, one has to have the measurability as in Definition 2.3. In Theorem 3.1, we prove that $\mathcal{G}_{p}(J)$ is non-void by showing that the family $\left\{\pi_{\Delta}(\cdot \mid J, \xi)\right\}_{\Delta \Subset \mathbb{Z}^{d}}$ possesses accumulation points, which are tempered Gibbs measures. Each measure of this kind is, therefore, obtained as the limit of $\left\{\pi_{\Delta_{n}}(\cdot \mid J, \xi)\right\}_{n \in \mathbb{N}}$ for the corresponding sequence $\left\{\Delta_{n}\right\}_{n \in \mathbb{N}}$ which, however, can depend on $J$ in an uncontrollable way (the so-called chaotic size dependenc ${ }^{3}$ ). In view of this fact, it is unclear whether these limiting points provide the measurability of $J \mapsto \mu(J)$. In Theorem 3.4. this measurability is obtained by means of general methods of the set-valued analysis. To the best of our knowledge, this is the first instance of the use of such methods in the theory of lattice models with random interactions.

- Theorems 3.6 and 3.7. These theorems give a constructive procedure of obtaining random Gibbs measures as the infinite volume limits. Even for $p=\infty$ and $q=1$, i.e. in the regular case of bounded interactions, Theorems 3.6 and 3.7 are the corresponding extensions of Theorems 6.2 .6 and 6.2 .8 in [1], p. 101-104. The novelty of these our theorems is that the chaotic size dependence is harnessed with the help of the Komlós theorem [10] - a renowned tool in the probability theory. This provides a new look at the approach put forward by C. M. Newman and D. L. Stein, see [1, 7, [8, 16] and the references therein.

- Theorems 3.8 and 3.9, For the translation invariant lattice systems with nonrandom interactions, the thermodynamic pressure exists and is unique even if the Gibbs measures are multiple, see Theorem 3.10 and Corollary 3.11 in [12], and/or Theorems 5.1.2 and 5.1.3 in [17. Thus, this is an important thermodynamic function by means of which one can establish, e.g., the absence/existence of phase transitions, see [19] and/or chapter 6 in [17]. For disordered systems, the pressure in $\Delta \Subset \mathbb{Z}^{d}$ clearly manifests the chaotic size dependence. For the model considered here, we propose to eliminate this effect by passing to the averages (3.7), as we did in Theorems 3.6 and 3.7 The existence of the limiting quenched pressure obtained in (3.12) is a generalization to unbounded spins of the relevant result of [13, 14]. The important point in Theorem 3.9] is that the pressure averaged over the disorder is the same

\footnotetext{
${ }^{2}$ See Definition 6.2.1 in [1].

${ }^{3}$ See the discussion in [8] and in [16], p. $55,56,64$.
} 
in all states, which resembles the corresponding fact known for nonrandom interactions, see Theorem 3.10 in [12] and Theorem 5.1.3 in [17], page 268. One observes that this result holds true for the Edwards-Anderson spin glass as well. For the systems of quantum anharmonic oscillators with the corresponding random interactions, the analogous statements can readily be proven by means of a combination of the methods of [12, 17] and those of the present work. This would be the extension of the results of [20].

\section{Acknowledgements}

The authors are grateful to Stas Molchanov and Michael Röckner for valuable discussions.

\section{References}

1. Bovier A., Statistical Mechanics of Disordered Systems. A Mathematical Perspective. Cambridge Series in Statistical and Probabilistic Mathematics. Cambridge University Press, Cambridge, 2006.

2. Aubin J.-P., Frankowska H., Set-valued Analysis. Reprint of the 1990 edition. Modern Birkhäuser Classics. Birkhäuser Boston, Inc., Boston, MA, 2009.

3. Lebowitz J.L., Presutti E., Comm. Math. Phys., 1976, 50, 195.

4. Ruelle D., Comm. Math. Phys., 1976, 50, 189.

5. Pasurek T., Theory of Gibbs Measures with Unbounded Spins: Probabilistic and Analytic Aspects. Habilitation Thesis, Universität Bielefeld, available as SFB 701 Preprint 08-101, 2008.

6. Georgii H.-O., Gibbs Measures and Phase Transitions. de Gruyter, New York, 1988.

7. Newman C.M., Stein D.L. Local vs. global variables for spin glasses. - In: Spin glasses, eds. E. Bolthausen and A. Bovier, p. 145-158, Lecture Notes in Math., 1900, Springer, Berlin, 2007.

8. Newman C.M., Stein D.L., Thermodynamic chaos and the structure of short-range spin glasses. In Mathematical aspects of spin glasses and neural networks, eds. A. Bovier and P. Picco, p. 243-287, Progr. Probab., 41, Birkhäuser Boston, Boston, MA, 1998.

9. Balder E.J., Probab. Th. Rel. Fields, 1989, 81, 185.

10. Komlós J. Acta Math. Acad. Sci. Hungar., 1967, 18, 217.

11. Aizenman M., Wehr J. Comm. Math. Phys., 1990, 130, 489.

12. Kozitsky Y., Pasurek T., J. Stat. Phys., 2007, 127, 985.

13. Contucci P., Lebowitz J., Ann. Henri Poincaré, 2007, 8, 1461.

14. Contucci P., Starr S., J. Stat. Phys., 2009, 135, 1159.

15. Parthasarathy K.R., Probability Measures on Metric Spaces. Probability and Mathematical Statistics, No. 3. Academic Press Inc., New York-London, 1967.

16. Newman C.M., Topics in Disordered Systems. Lectures in Mathematics ETH Zürich. Birkhäuser Verlag, Basel, 1997.

17. Albeverio S., Kondratiev Y., Kozitsky Y., Röckner M. The Statistical Mechanics of Quantum Lattice Systems. A Path Integral Approach. EMS Tracts in Mathematics, 8. European Mathematical Society (EMS), Zürich, 2009.

18. Kondratiev Y., Kozitsky Y., Pasurek T., J. Appl. Prob., 2010, 47, 856.

19. Kargol A., Kondratiev Y., Kozitsky Y., Rev. Math. Phys., 2008, 20, 529.

20. Contucci P., Lebowitz J., J. Math. Phys., 2010, 51, 023302. 


\title{
Стани Ґіббса ґраткових спінових систем з необмеженим безладом
}

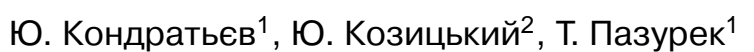

1 Факультет математики, Університет Білефельд, D-33615, Німеччина

2 Інститут математики, Університет Марії Кюрі-Склодовської, 20-031 Люблін, Польща

Досліджуються стани Ґіббса спінової системи на ґратці $Z^{d} 3$ парною взаємодією $J_{x y} \sigma(x) \sigma(y)$. Тут $\langle x, y\rangle \in \mathrm{E}$, тобто $x$ та $y$ є сусідами в $Z^{d}$. Інтенсивності $J_{x y}$ і спіни $\sigma(x), \sigma(y)$ приймають довільні дійсні значення. Щоб контролювати ї ріст, ми вводимо відповідні множини $\mathcal{J}_{q} \subset \mathbb{R}^{\mathrm{E}}$ і $\mathcal{S}_{p} \subset \mathbb{R}^{\mathbb{Z}^{d}}$ та показуємо, що для кожного $J=\left(J_{x y}\right) \in \mathcal{J}_{q}$ : (а) множина станів Ґіббса $\mathcal{G}_{p}(J)=\left\{\mu:{ }^{\prime}, \mu\left(\mathcal{S}_{p}\right)=1\right\}$ $€$ непорожньою і компактною в слабкому сенсі; (b) кожна $\mu \in \mathcal{G}_{p}(J)$ задовільняє інтегральній оцінці, однаковій для всіх $\mu$. Далі ми досліджуємо випадок, коли на $\mathcal{J}_{q}$ вводиться норма, борелевське $\sigma$ тіло $\mathcal{B}\left(\mathcal{J}_{q}\right)$ та повна ймовірнісна міра $\nu$. Ми показуємо, що мультивідображення $\mathcal{J}_{q} \ni J \mapsto \mathcal{G}_{p}(J)$ має вимірні селектори $\mathcal{J}_{q} \ni J \mapsto \mu(J) \in \mathcal{G}_{p}(J)$ - суть випадкової міри Ґіббса. Ми показуємо, що емпіричні розклади $N^{-1} \sum_{n=1}^{N} \pi_{\Delta_{n}}(\cdot \mid J, \xi)$, одержані з локальних умовних мір Ґіббса $\pi_{\Delta_{n}}(\cdot \mid J, \xi)$ та 3 вичерпуючих послідовностей $\Delta_{n} \subset \mathbb{Z}^{d}$, мають $\nu$ - майже певні границі при $N \rightarrow+\infty-$ суть випадкової міри Ґіббса. Подібним чином ми показуємо існування $\nu$ - майже певних границь емпіричних метастанів $N^{-1} \sum_{n=1}^{N} \delta_{\pi_{\Delta_{n}}(\cdot \mid J, \xi)}$, які $€$ метастанами Айзенмана-Вера. Накінець, ми показуємо існування граничного термодинамічного тиску за певних додаткових умов на $\nu$.

Ключові слова: метастан Айзенмана-Вера, емпіричний метастан Ньюмена-Штайна, хаотична просторова залежність, теорема Комлоша, спінове скло 